Edmundo Pimentel 1

\title{
El desarrollo sostenible de los sistemas de salud: un paradigma emergente y saludable
}

\author{
The sustainable development of the systems of health: \\ an emergent and healthy paradigm
}

1 Universidad Central de Venezuela. Ciudad Universitaria, Caracas, Venezuela. CONAFIN, S.A. Calle Los Laboratorios, Torre Beta, Piso 4, Oficina 407. Los Ruices, Caracas 1071. edipime@cantv.net
A bstract During the last decades several countries all over the world, specially Latin-Americans, have engage themsel ves in actions to reforms their health care systems, without reaching the expected quality life for its inhabitants life, in spite of the alarming social expenses. Governments of these countries have tried to improve conditions of life, increasing social expenses up to the maximum of their possibilities, without having obtained the expected results. For these reasons, it is gaining many adepts, in the academic debate, the idea of improving efficiency in the provision of social services, in a framework of private and Government participation, that will ensure equity and private capital participation, in order to introduce substantial changes at different levels, with the objective of improving equity of ben efits, management efficiency and effectiveness of its services to satisfy health care needs of the population. The final objective is to contribute to improve health and living conditions of the population, reducing inequalities and improving service levels. For this purpose it is necessary to modernize public institutions, establish new relations among the different players, a new balance between public and private sector and the required sustainable financial model. Key words Efficiency, Reforms, $\mathrm{H}$ ealth's systems, Sustainable model
Resumen Durante lasúltimas décadas, países de todo el mundo, y en especial los latinoamericanos, han emprendido acciones de reformas a sus sistemas de salud, impulsados por el crecimiento del gasto social. Los gobernantes han tratado de mejorar las condiciones de vida, impulsando el gasto social hasta el máximo de sus posibilidades sin haber obtenido los resultados esperado. Los desafíos recientes de la globalización y apertura económica han aumentado las restricciones de los gobiernos para financiar políticas públicas. Las reformas del sector salud son procesos orientados a introducir cambios sustantivos en diferentes instancias, en sus relaciones y en las funciones que realizan, con el propósito de aumentar la equidad de sus beneficios, la eficiencia de su gestión y la efectividad de sus prestaciones para sati ffacer las necesidades de salud de la población. El objetivo es contribuir a mejorar las condi ciones de vida y salud de la personas, reduciendo desigualdades y mejorando la atención. Para ello se debe emprender la modernización de las instituciones públicas, la búsqueda de nuevas relaciones entre actores, un nuevo equilibrio entre lo público y lo privado y la necesaria sostenibilidad financiera.

Palabras-claves Eficiencia, Reformas, Sistemas de salud, Sostenibilidad 
La salud es un punto de confluencia de lo económico y social, de lo individual y lo comunitario, de lo público y lo privado, de la eficiencia y la equidad, del conocimiento y la acción, de la tecnología y la cultura. Existe una relación simbiótica entre salud y economía, ambas se retroalimentan positivamente: un mayor grado de desarrollo económico influirá positivamente en mayores niveles de salud y viceversa.

Los servicios de salud se relacionan con la economía desde dos planos diferentes. Por una parte constituyen un componente fundamental del desarrollo y bienestar social (los progresos en salud y educación significan acumulación de capital humano), y por la otra representa una de las principales fuentes de empleo, que demanda grandes cantidades de insumos y genera importantes innovaciones tecnológicas.

Durante varias décadas el concepto de desarrollo de la salud estuvo vinculado al postulado que un mayor crecimiento económico generaría mayores recursos para ese sector. Esta percepción derivada de la controvertida Teoría del Derrame, que sostuvo que el crecimiento económico derramaría sus ben eficios sobre otros sectores impulsando su desarrollo, fue una de las causantes que durante varias décadas el sector no percibiese las inversiones que requería.

Al constatarse que en los últimos cincuenta años la economía mundial tuvo el mayor crecimiento registrado en la historia de la humanidad y que sus beneficios no se derramaron hacia los otros sectores de la sociedad como se esperaba, que por el contrario, la pobreza y la inequidad también crecieron a pasos agigantados, muchos gobernantes trataron de subsanar sus errores aumentando al máximo de sus posibilidades las asignaciones presupuestarias para el desarrollo del capital humano, sin preocuparse de implantar medidas que contribuyese a rentabilizar socialmente esa inversión.

El postulado, en el que se afirma que con solo aumentar las asignaciones presupuestarias al sector salud este mejoraría sustancialmente, fue una de las causas que originó el incontrolado y creciente gasto, que hoy preocupa a los gobernantes de todos los países del mundo, desde los más pobres hasta los más industrializados.

Es obvio que la disponibilidad de recursos económicos constituye uno de los pilares fundamentales del desarrollo de la salud, no obstante, los hechos han demostrado que es una condición necesaria pero no suficiente, al respecto debemos recordar la afirmación de Amartya Sen: Tan estúpido sería pretender que una renta elevada no es un factor que favorecela buena salud y la supervivencia prolongada, como sostener que es el único factor que contribuye.

La siguiente reseña es un ejemplo abrumador de la afirmación de Sen: en 1990 los Estados U nidos de N orteamérica destinaron a la salud el $12,7 \%$ de su PIB lo cual equivalió a unos 690 mil millones de dólares, cifra similar al PIB del Reino U nido, la séptima potencia más grande del mundo.

En Latinoamérica entre 1990 y 1999 el gasto social promedio de la región fue incrementado en un 50\%, al pasar de 360 a 540 dólares anuales per capita y el porcentaje de población en condiciones de pobreza, disminuyó levemente al pasar de $48,3 \%$ en 1990 a 43,8\% en 1999 . No obstante está proporción resultó ligeramente superior a la registrada en 1980 (40,5\%). En términos absolutos, el número de personas que viven bajo condiciones de pobreza aumentó en 64,3 millones al pasar de 135,9 millones en 1980 a 200,2 millones en 1999 (O campo, 2001).

Sin embargo, a pesar de estos esfuerzos, los resultados son desalentadores. Los problemas de pobreza y desigualdades que estuvieron en el origen de estas medidas de reforzamiento de las políticas sociales siguen presentes, en mayor o menor grado, en los países latinoamericanos. No solo la conocida pobreza einequidad, sino que surgen además nuevas problemáticas sociales que, en muchos casos, agravan las situaciones originales ( $\mathrm{Ar}$ dí, 2002).

La situación es más grave y desalentadora para aquellos países con bajos ingresos, donde viven unas 2.400 millones de personas cuya esperanza de vida se ubica en 55 años y donde de cada mil niños mueren cerca de 140 antes de cumplir los cinco años.

En esos países pobres, el gasto anual per capita en salud se ubica en 21 dólares y en los países ricos en más de 2.000. Un estudio realizado por una comisión de economistas y científicos convocados por la Organización M undial de la Salud (OMS) revela que si los países ricos destinaran el 0,1 por ciento más de su producto bruto para ayuda en salud a esos países de bajos ingresos, se salvarían anualmente ocho millones devida.

En un artículo titulado "La gran mentira del gasto social y la dichosa redistribución", el analista mexicano Adolfo Gutiérrez Chávez plantea que En 1990, el gasto social representaba al rededor del $38 \%$ del gasto total, lo que si gnifica que el gobierno destinaba 38 centavos de cada peso que gastaba para programas sociales. En el año 2000, 
este gasto aumentó a 62 centavos. Así, con el propósito de sacar a millones de familias de la marginación, y con ello aliviar las condiciones de pobreza, el gobierno ha incrementado el gasto social cerca de $114 \%$ en los últimos diez años. Hace diez años, la quinta parte de los hogares más pobres obtenían cinco pesos de cada cien que se generaban en el país. A hora obtienen menos de cuatro. La pregunta obvia: ¿dónde quedaron los 24 centavos que el gobierno nos quitó de más para aliviar la pobreza? En términos de eficiencia la calificación del gasto social es sin lugar a dudas reprobatoria.

El articulista mexicano continúa su disertación preguntándose: ¿Qué pasó con los recursos que el gobierno nos quitó de más para combatir la pobreza? Pues dado que la pobreza no sólo no disminuyó sino que aumentó, lo que no regresó a las familias de más altos ingresos se perdió en algún rinconcito del laberinto de la burocracia.

Finalmente concluye su artículo con los siguientes planteamientos: Incrementar el gasto social no sirve de nada porque o es regresivo o se lo traga la administración pública o se lo roba algún vival, así que, entonces, ¿quéhacemos con él? Una alternativa podría ser entregar los recursos de esa gran bolsa en forma directa a las familias más necesitadas.

La experiencia de América Latina y de los demás países, continua Adolfo Gutiérrez Chávez, demuestra que ni el gasto social ni los programas sociales funcionan para salir de la pobreza y alcanzar el desarrollo. Lo único que nos puede ayudar a dejar de ser pobres y alcanzar mejores condiciones de vida es el crecimiento económico que, sin distorsiones causadas por la intervención gubernamental, genera oportunidades para todos.

La situación descrita en el artículo comentado, que analiza el gasto social mexicano, tiene su correlato el la mayoría de los países latinoamericanos, donde el gasto social creció sustancialmente durante los últimos diez años sin que se haya logrado disminuir la pobreza ni las inequidades existentes en la región. Este hecho ha inducido a que se extraigan conclusiones equívocas como las del articulista, que propicien a retomar los postulados de la fracasada Teoría del Derrame.

Los gobiernos del mundo comparten el temor que el creciente gasto en salud termine escapándoseles de las manos, lo cual ha propiciado un tema emergente: "M ejorar la eficiencia del gasto en salud".

El crecimiento sostenido de los gastos en salud sin que se perciban las mejorías esperadas en el sector, ha impulsado a los gobernantes de casi todos los países del mundo, sin importar su nivel de desarrollo, a promover constantes reformas de sus sistemas de salud, en una búsqueda de mayor eficiencia y eficacia que garanticen una mayor calidad, cobertura y equidad.

En la última década los países desde Estados U nidos y M éxico hasta Costa Rica, desde CoIombia y Venezuela hasta Chile, desde Gran Bretaña, H olanda, Suecia y España, hasta China, se han visto afectados por los vientos de cambio que soplan por los cuatro puntos cardinales del Orbe.

La nueva visión de las políticas sociales puede ser considerada como un tema emergente. Los desafíos recientes de la globalización y apertura económica han aumentado las restricciones de los gobiernos para financiar políticas públicas. De esta manera, ha comenzado a ganar adeptos en el debate académico la idea de mejorar la eficiencia en la provisión de los servicios sociales por parte del Estado en un marco de gestión mixta, que asegure la equidad y la participación de las empresas privadas.

Para 1997 la CEPAL había identificado a 17 países de América Latina que habían emprendido reformas en sus sistemas de salud. Algunas de esas reformas pretenden remover el sistema en su totalidad, otras son más parciales pero todas introducen modificaciones importantes con respecto a lo que existía anteriormente. La mayoría de dichas reformas se encontraban en una fase inicial o habían sido enunciadas pe ro no ejecutadas. En la mayoría de los países de la región, estas reformas se basaron en aspectos distintos a la búsqueda de la eficiencia del sector salud y mostraban diferencias importantes con las implantadas en países desarrollados.

Las reformas adoptadas por algunos países desarrollados pretenden mejorar la eficiencia de la asignación de los recursos promoviendo el acceso a los servicios con equidad. Asimismo se busca la eficiencia de las empresas a través del mecanismo de elección del proveedor por parte de los usuarios y un nivel suficiente de autonomía en la gestión de los prestadores, y como re quisito un adecuado nivel de eficacia y efectividad en las decisiones clínicas. Pero estas medidas son suficientes.

Las reformas del sector salud son procesos orientados a introducir cambios sustantivos en diferentes instancias, en sus relaciones y en las funciones que realizan, con el propósito de aumentar la equidad de sus beneficios, la eficiencia de su gestión y la efectividad de sus presta- 
ciones para satisfacer las necesidades de salud de la población. El objetivo final es contribuir a mejorar las condiciones de vida y salud de las personas, reduciendo desigualdades y mejorando la atención. Para ello se debe emprender la modernización de las instituciones públicas, la búsqueda de nuevas relaciones entre actores, un nuevo equilibrio entre lo público y lo privado y la necesaria sostenibilidad financiera. Dicho en pocas palabras, se requiere un Plan de Gestión Sustentable cuyas bases deben ser las siguientes: información y comunicación, educación y conciencia pública, participación ciudadana, descentralización y desconcentración administrativa, recursos humanos, y coordinación intersectorial.

\section{Información y comunicación}

El uso de la información va más allá de la diseminación de contenidos generales. El desafío que supone modificar las prácticas sociales para mejorar la eficiencia y eficacia de los sistemas de salud así como garantizar su calidad y equidad es muy amplio, los caminos para alcanzar la sostenibilidad no son claros ni uniformes, las alianzas que hay que construir significa contar con muchos actores con distintas disciplinas y culturas y con desconfianzas y resistencias difíciles de vencer.

Una estrategia eficaz para superar los problemas derivados de la diversidad de actores, interese, valores y deseos, es asegurar desde el inicio una información clara, sencilla, oportuna, accesible y confiable. AI margen de la necesidad de información de los interesados en actuaciones concretas, está la información de los servicios de salud, a la que debe tener acceso el público en general.

La comunicación entraña un paso más en el uso de los instrumentos sociales, en la que se trata de buscar respuestas del interlocutor como medio válido para ajustar el mensaje y asegurarse que sea entendido. La comunicación es un proceso de interacción social que capacita a la comunidad para entender los factores claves y sus interdependencias y para actuar sobre los problemas en forma competente.

El esfuerzo que se ha realizado con el fin de dar a conocer las opciones de los sistemas de salud en sus distintos niveles asistenciales y las oportunidades para su utilización sostenible, en el nivel educativo tanto formal como no formal, es pequeño y no alcanza a toda la población, se realiza en forma desarticulada y responde a diferentes iniciativas e intereses. No se atienden en toda su magnitud las demandas de las comunidades por programas de educación integrales que den a conocer la necesidad de buscar opciones viables de participación, supervisión y fiscalización de los programas de salud pública. Como resultado, la sociedad actual no valora aún adecuadamente los niveles primarios de asistencia médica ni la potencialidad de su participación.

\section{Educación y conciencia pública}

La educación a la que se refiere la estrategia es un proceso social que rebasa el ámbito educativo formal. Se trata de una educación dirigida a evitar y solucionar los problemas de la salud, especialmente en su fase preventiva, concebida como un instrumento de gran potencial para impulsar la participación ciudadana como elemento fundamental en el logro de la eficiencia y eficacia de la gestión pública.

En la educación formal, las reformas sectoriales de salud representan un gran desafío para las universidades, específicamente en la formación de profesionales dotados de nuevas competencias, que le permita integrar equipos multidisciplinarios y multiculturales enfocados hacia el uso eficiente de los recursos que le son asignados al sector. Las universidades deberán mejorar la formación de esto profesionales mediante el diseño de modelos de intervención acordes con las nuevas realidades y los procesos de integración subregional y globalización, la ampliación de la extensión universitaria, la actualización de sus conocimientos y la investigación aplicada.

Es una responsabilidad de la universidad asegurar la actualización del profesional. Ello implica el desarrollo efectivo de la educación continua y permanente en estrecha articulación con los ministerios de salud, los servicios, los colegios profesionales y las asociaciones científicas. Esta nueva responsabilidad universitaria consolidará la integración con los servicios y contribuirá a su adecuación y pertinencia.

\section{Participación ciudadana}

La participación es la estrategia fundamental para obtener los cambios que se requieren, en los sistemas de salud. Solo a través de la partici- 
pación se puede crear la cohesión social necesaria para resolver los complicados problemas a los que se enfrenta nuestra sociedad.

La participación requiere de un aprendizaje. Se trata de una profundización democrática en la que se deben desarrollar competencias para la acción, mediante el aprendizaje y reforzamiento de procedimientos, además de anima a las personas para que se sientan con el ánimo necesario para comprometerse organizadamente en la acción.

Una de las formas más eficiente de lograrlo es propiciar la participación directa en programas locales de salubridad y en campañas epidemiológicas, que puedan suministrar criterios de evaluación y seguimiento para la toma de decisiones consensuadas, que constituyan experiencias gratificantes.

\section{La descentralización y desconcentración administrativa}

La visión tradicional de las políticas sociales tenía incorporada una idea de Estado centralista, a la vez financista y proveedor de los servicios sociales. La nueva visión introduce la descentralización como un elemento central en el análisis del diseño de las distintas políticas. Este concepto implica que los organismos centrales de decisión renuncian a parte del poder que poseen en la fijación y monitoreo de las políticas sociales, transfiriéndolos a los ámbitos locales de decisión y reservando al gobierno central la tarea de dictaminar las directivas globales.

La idea implícita es que al transferir responsabilidades a los gobiernos locales, o unidades ejecutoras locales, aproveche la proximidad de éstos con los usuarios de los servicios públicos para producir un efecto de mayor eficiencia en la forma en que serán entregados. El grado de autonomía que adquieren los decisiones locales retroalimentan las políticas implementadas ya que al conocer las características del lugar y de las personas que lo habitan, la oferta de los servicios sociales se hace más acorde a las necesidades y peculiaridades locales. De esta manera se supera una de las principales críticas de burocratismo y verticalismo que se realizaba a los programas altamente centralizados.

La última década ha sido particularmente rica en experiencias que han buscado profundizar la descentralización y la desconcentración de la gestión pública en sus diferentes ámbitos. En el campo de la política social, al delegar el go- bierno central, por la vía de la descentralización, responsabilidades antes ejercidas por él hacia los gobiernos subnacionales (estados o provincias y municipios), y transferir recursos y las facultades para tomar decisiones sobre su asignación, ha pretendido lograr diversos objetivos, de gestión, financieros y políticos. Entre ellos vale resaltar, en primer término, la adecuación de los programas sociales a las demandas y singularidades locales, así como aumentar el impacto y la eficiencia de la política y el gasto público sociales; entre otros factores, las acciones intersectoriales e integrales tendrían que resultar mucho más viables a nivel local, dadas las menores escal as. En segundo lugar, se pretende potenciar los recursos propios de las localidades.

Finalmente, se busca profundizar la democracia al acercar el gobierno a los ciudadanos y propiciar una mayor participación ciudadana; esta vinculación más estrecha entre autoridades y población debe generar un movimiento integrador y dinámico de participación y control en la prestación de los servicios y favorecer la comunicación y el flujo de información más expeditos entre decisores, administradores y destinatarios.

El principal problema que han enfrentado la mayoría de los procesos de descentralización implantados es la falta de capacidades técnicas existentes a nivel regional. Se supone que con el avance del proceso, los individuos más capacitados se verán atraídos hacia los puestos de dirección locales, por las buenas perspectivas profesionales y económicas, pero esto puede tardar lo suficiente como para hacer fracasar el proceso por incapacidad técnica. Es así que todo buen proceso de descentralización de los servicios sociales debe preocuparse por capacitar a las personas que se constituirán en los líderes del cambio.

Descentralizar no generará, por el hecho mismo de hacerlo, un efecto más positivo sobre la provisión del servicio social que si éste se hiciese en forma centralizada. Se deben tener en cuenta las particularidades de cada situación, las capacidades regionales, la cultura democrática de cada país y el diseño de los programas de transferencias desde las órbitas centrales a las locales.

Las principales lecciones y retos de la descentralización en el ámbito de la política social se relacionan con la organización institucional y la eficiencia, los balances fiscales, la equidad y la participación social. Estos temas se entrecruzan, como es obvio, con aspectos más generales 
asociados al proceso de descentralización en su conjunto. En los casos más recientes se observan algunos cambios positivos, como los esfuerzos por alcanzar un consenso respecto de la adecuada secuencia para la puesta en marcha de estas reformas y el rediseño de los sistemas de transferencias, en forma más acorde con los objetivos de eficiencia y equidad regional.

\section{Recursos humanos}

El Plan de Gestión Sustentable debe ser acompañado de un programa de promoción del recurso humano relacionado con la gestión de salud en los ámbitos estatal y privado; no solo en lo concerniente a los planes de acción, sino que además debe incluirse el uso de la infraestructura que poseen las instituciones dispensadoras de salud, tanto públicas como privadas. Existe un distanciamiento entre quienes poseen el conocimiento y la capacidad de transmitirlo, y quienes son responsables del manejo del recurso.

H asta el presente no se ha producido la integración requerida entre el sector público y el sector privado para enfrentar las limitaciones impuestas al crecimiento del aparato estatal y las posibilidades de control y desarrollo de los recursos de la salud.

A finales de 1997, en la ciudad de San Joséde Costa Rica, bajo los auspicios del Gobierno de Costa Rica y la O rganización Panamericana de la Salud (OPS-OMS), se realizó la Reunión Regional Recursos Humanos, bajo el eslogan: Factor Crítico de la Reforma Sectorial en Salud. El propósito de la reunión fue llamar la atención de los líderes del sector salud de la región, sobre la importancia estratégica de los recursos humanos para el logro de los objetivos y el avance de los procesos de reforma de los sistema de salud.

La gran enseñanza que quedó de esa reunión, es que el cambio en salud se hará con la gente que ya está, con el personal existente. Ese personal es el gran capital para cambiar. Reconocido este hecho, la estrategia se debe enfocar hacia como generar condiciones favorables y estables para asegurar la participación del perso- nal en ese cambio. Esta importante conclusión muestra la necesidad de emprender un gran programa de educación, en el que se ponga a la capacitación del personal del sector como instrumento fundamental del cambio. El mensaje es muy claro: el cambio en los procesos de reforma sectorial empezará cuando los recursos humanos lo asuman como suyo.

Los procesos de reforma del sector, en su mayor proporción, alcanzarán el éxito solamente si los recursos humanos se comprometen en la búsqueda de los fines de la reforma, buscando la universalidad en el acceso y la atención y la equidad del sistema. Ello supone que el personal tenga flexibilidad para aceptar el cambio, el conocimiento, las habilidades, las destrezas requeridas, así como una clara comprensión y ubicación del papel quejuegan para cuidar y preservar de manera integral la salud de las comunidades.

\section{Coordinación intersectorial}

En virtud de la complejidad, de la diversidad y de lo atomizada que es la gestión que le corresponde acometer a los sistemas de salud, es fundamental que focalicen sus esfuerzos y comprometan una parte importante de sus recursos en mejorar la coordinación con otras instituciones privadas y de la Administración Pública Nacional.

Todavía no existe una integración efectiva de los diferentes sectores para la gestión sostenible de la salud. Para la mayoría resulta un tema muy nuevo, y se observa mayor apropiación del tema por parte de la sociedad civil, no directamente responsable de la gestión de salud, que dentro de instituciones del Estado. Los espacios con que cuentan las instituciones del Estado para la planificación, coordinación, participación y corresponsabilización de la gestión de salud con la sociedad civil, aún no se han consolidado.

Se carece de los instrumentos (normativa, convenios, etc.) e instancias formales ya en funcionamiento que permitan un abordaje integral del tema de la salud y uso sostenible de los recursos asignados. 
Figura 1

Un modelo de gestión sostenible para el sector salud

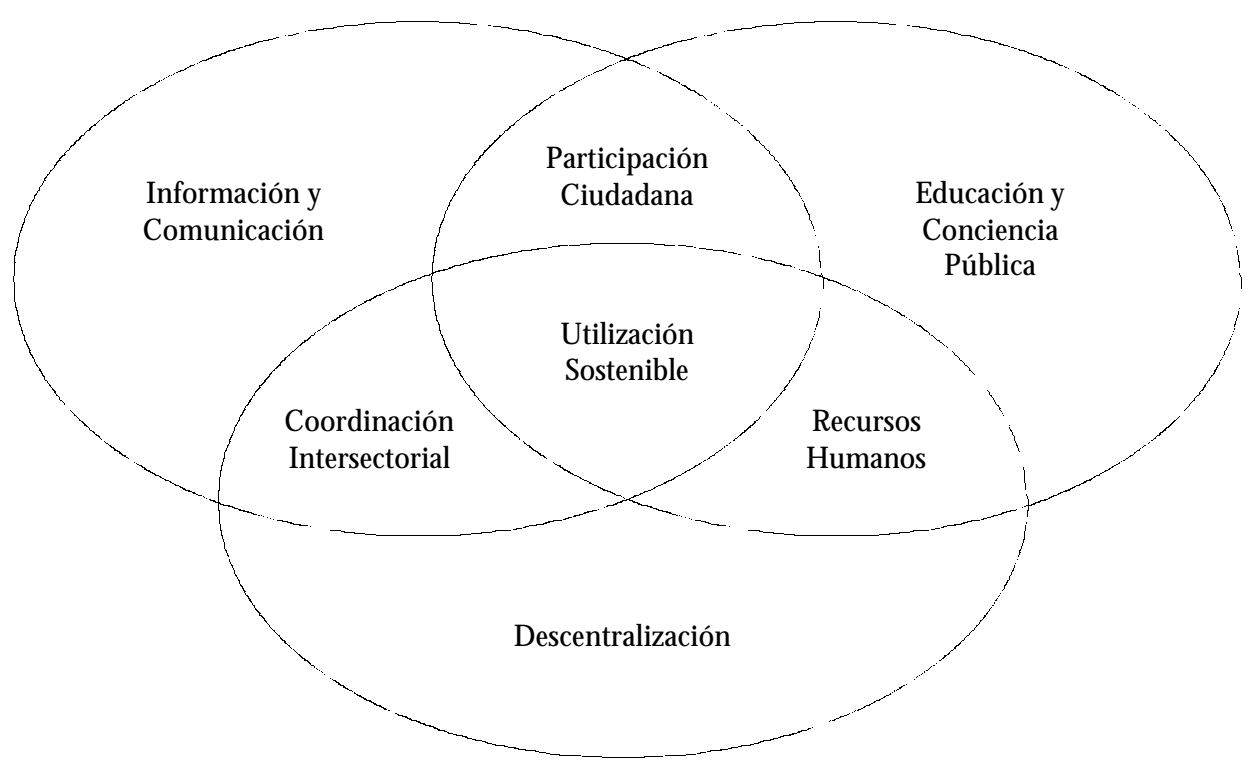

\section{Referências bibliográficas}

Ardí C 2002. Una nueva generación de reformas sociales en América Latina. Colección I deas 17. Chile

Banco M undial 2000. En el umbral del siglo XXI. Banco M undial, Washington.

CEPAL 2000. Equidad, desarrollo y ciudadanía. Washington $D C$.

Desai M 1992. Bienestar y pobreza: un índice de progreso social. Calidad de vida. FCE, M éxico

Geertz C 1983. Descripción densa: hacia una teoría interpretativa de la cultura. Gedisa, Barcelona.

Gómez JA 1998. La calidad de vida y el Tercer Sector: nuevas dimensiones de la complejidad. FCE, M éxico.

Gryspan R 2000. La pobreza en América Latina y estrategias para superarla. Seminario Internacional U nesco, FLACSO, San José de Costa Rica.

Kliksberg B 2000. América Latina: una región en riesgo, pobreza, inequidad e institucionalidad social. BID, Washington, DC.

Kliksberg B 2001. El capital social. Editorial Panapo, Caracas.
Kliksberg B \& Tomassini L (comps.) 2000. Capital social y cultura: claves estratégicas para el desarrollo. BID/ FCE, M éxico.

Kliksberg B (comp.) 1997. Pobreza. Un tema impostergable. (4a ed.). Fondo de Cultura Económica, M éxico.

Lecture P 1998. Towards a new paradigm for development: strategies, policies and processes. UNCTAD, Geneva.

N ussbaum M C \& Sem A (comps.) 1996. La calidad de vida. Fondo de Cultura Económica, M éxico.

O campo JA 2001. Panorama social de América Latina. CEPAL, $N$ aciones U nidas.

PNUD 1992. El abismo de la desigualdad. Informe Sobreel Desarrollo H umano. PNUD, Washington DC.

PNUD 2000. Informe Sobre D esarrollo H umano. PNUD, Washington DC.

Stiglitz JE 1998. M ás instrumentos y metas más amplias para el desarrollo. Hacia el consenso Post-W ashington. CEPAL/ECLAD, San José de Costa Rica.

Artigo apresentado em 23/8/2002

Aprovado em 25/10/2002

Versão final apresentada em 5/12/2002 